\title{
Glomerular parietal epithelial cells in kidney physiology, pathology, and repair
}

\author{
Stuart J. Shankland ${ }^{\mathrm{a}}$, Hans-Joachim Anders ${ }^{\mathrm{b}}$, and Paola Romagnanic
}

\begin{abstract}
Purpose of review
We have summarized recently published glomerular parietal epithelial cell (PEC) research, focusing on their roles in glomerular development and physiology, and in certain glomerular diseases. The rationale is that PECs have been largely ignored until the recent availability of cell lineage tracing studies, human and murine PEC culture systems, and potential therapeutic interventions of PECs.
\end{abstract}

\section{Recent findings}

Several new paradigms involving PECs have emerged demonstrating their significant contribution to glomerular physiology and numerous glomerular diseases. A subset of PECs serving as podocyte progenitors have been identified in normal human glomeruli. They provide a source for podocytes in adolescent mice, and their numbers increase in states of podocyte depletion. PEC progenitor number is increased by retinoids and angiotensin-converting enzyme inhibition. However, dysregulated growth of PEC progenitors leads to pseudo-crescent and crescent formation. In focal segmental glomerulosclerosis, considered a podocyte disease, activated PECs increase extracellular matrix production, which leads to synechial attachment and, when they move to the glomerular tuft, to segmental glomerulosclerosis. Finally, PECs might be adversely affected in proteinuric states by undergoing apoptosis.

\section{Summary}

PECs play a critical role in glomerular repair through their progenitor function, but under certain circumstances paradoxically contribute to deterioration by augmenting scarring and crescent formation.

\section{Keywords}

crescent, glomerulosclerosis, podocyte, progenitors, regeneration

\section{INTRODUCTION}

The urinary space of the glomerulus is lined by Bowman's basement membrane (commonly called Bowman's capsule), upon which attaches a monolayer of glomerular parietal epithelial cells (abbreviated herein as PECs). In this regard, PECs are similar to the kidney's other epithelial cell monolayer, namely, podocytes and tubular epithelial cells. Morphologically, PECs resemble squamous epithelial cells, with a small cell body size ranging in thickness from 0.1 to $0.3 \mu \mathrm{m}$, increasing to 2.0-3.5 $\mu \mathrm{m}$ at the nucleus. Scanning electron microscopic studies show that PEC surfaces are lined by microvilli and cilia ( $0-2$ cilia per cell). The functions of these are not known in this cell type. The number of PECs in a normal rat glomerulus is estimated to comprise $14.8 \%$ of all glomerular cells. Despite their thin cell bodies, PECs form junctions between adjacent cells, comprising a very complicated and delicate structure, described as 'labyrinth-like.'
Transmission electron microscopic studies show tight junctions near the apical surface of PECs [1].

The study of PECs in health and disease is increasingly being recognized through vigorous studies by several investigators globally. This has been enabled by the generation of primary and immortalized PECs in culture [2,3], the establishment of reporter mice to cell fate map PECs [4], the

\footnotetext{
aDivision of Nephrology, University of Washington Medical Center, Seattle, Washington, USA, ${ }^{b}$ Division of Nephrology, Medizinische Klinik und Poliklinik IV, Klinikum der Universität München-Innenstadt, Munich, Germany and ${ }^{c}$ Excellence Centre for Research, Transfer and High Education for the Development of De Novo Therapies (DENOTHE), University of Florence, Florence, Italy

Correspondence to Stuart J. Shankland, MD, MBA, 1959 NE Pacific Avenue, Box 356521, Room BB1269, Seattle, WA 98195-6521, USA. Tel: +1 206543 2346; fax: +1 206685 8661; e-mail: stuartjs@ u.washington.edu
}

Curr Opin Nephrol Hypertens 2013, 22:302-309

DOI:10.1097/MNH.0b013e32835fefd4 


\section{KEY POINTS}

- Glomerular PECs serve as progenitors for terminally differentiated podocytes in healthy glomeruli, and following a decrease in podocyte number in disease.

- Dysregulated PECs lead to crescent and pseudocrescent formation in crescentic and collapsing glomerulopathies, respectively.

- Several interventions have been shown to regulate PECs' progenitor function.

- In FSGS, activated PECs produce extracellular matrix proteins that form the basis for the synechial attachments and segmental glomerulosclerosis.

- Tight junction proteins between adjacent PECs likely serve to limit filtered protein to the urinary space.

identification of proteins and genes expressed by these cells in normal and diseased experimental and human conditions, and the application of molecular and cellular approaches that allow researchers to begin to probe how these cells may respond to certain interventions. The purpose of this review is to provide a recent literature update of our current understanding of how PEC biology underlies normal function in health (Table 1), and how in glomerular diseases PECs may serve a critical reparative role, or under different circumstances the response by PECs may lead to further glomerular damage.

\section{PARIETAL EPITHELIAL CELLS AS THE GOOD GUYS IN DEVELOPMENT, HEALTH, AND DISEASE}

Studies show that PECs are regarded as 'good guys' as they play vital roles during normal glomerular development, and have critical biological functions required for normal function in states of health and disease.

\section{Role in normal glomerular development}

During nephrogenesis, PECs develop from the metanephric mesenchyme that is induced by the ureteric bud to gradually acquire an epithelial phenotype and form the first stage of nephron development, the renal vesicle, which undergoes a series of invaginations and elongations to generate the S-shaped bodies [5]. At this stage, the middle and distal segments of the S-shaped body that remained in contact with the ureteric bud epithelium fuse to form a single, continuous epithelial tube. The proximal end is invaded by blood vessels, and Bowman's space begins to form [5]. During development from the S-shaped body to the capillary loop stage, some PECs constitute Bowman's capsule, whereas others upregulate podocyte-specific genes and begin the de-novo expression of the cyclin-dependent kinase inhibitor p27, and downregulation of PAX2, to differentiate into podocytes [5]. The downregulation of PAX2 by WT1 seems to be a prerequisite that permits this WT1-controlled differentiative process [6] (Fig. 1). WT1 is a powerful inhibitor of

Table 1. Role of parietal epithelial cells in health and disease

\begin{tabular}{lll} 
Functional role in healthy glomerulus & Reparative role of PECs in disease & $\begin{array}{c}\text { Abnormal pathobiology contributes } \\
\text { to damage in disease }\end{array}$ \\
\hline Progenitor function for podocytes & $\begin{array}{c}\text { PEC progenitors differentiate into } \\
\text { podocytes, possibly replacing podocytes } \\
\text { decreased in disease }\end{array}$ & $\begin{array}{c}\text { Fibrotic attachment and segmental } \\
\text { sclerosis }\end{array}$ \\
$\begin{array}{c}\text { Intracellular tight junctions restrict } \\
\text { glomerular filtrate to urinary space }\end{array}$ & $\begin{array}{c}\text { An uncontrolled/dysregulated proliferation } \\
\text { of PECs and the PEC progenitor } \\
\text { subpopulation leads to excess PEC } \\
\text { number, forming crescents and } \\
\text { pseudo-crescents }\end{array}$ \\
$\begin{array}{c}\text { Possible synthesis and repair of } \\
\text { Bowman's basement membrane }\end{array}$ & $\begin{array}{c}\text { Decrease in tight junction protein expression } \\
\text { accompanied by leakage of glomerular } \\
\text { filtrate into periglomerular space }\end{array}$ \\
& $\begin{array}{c}\text { Excess albumin uptake leads to apoptosis, } \\
\text { leading to reduced progenitor pool }\end{array}$ & $\begin{array}{c}\text { Expression pattern consistent with } \\
\text { epithelial-to-mesenchymal transition }\end{array}$ \\
& $\begin{array}{l}\text { Growth factor production and changes in } \\
\text { levels of growth factor receptors }\end{array}$
\end{tabular}

PEC, parietal epithelial cell. 


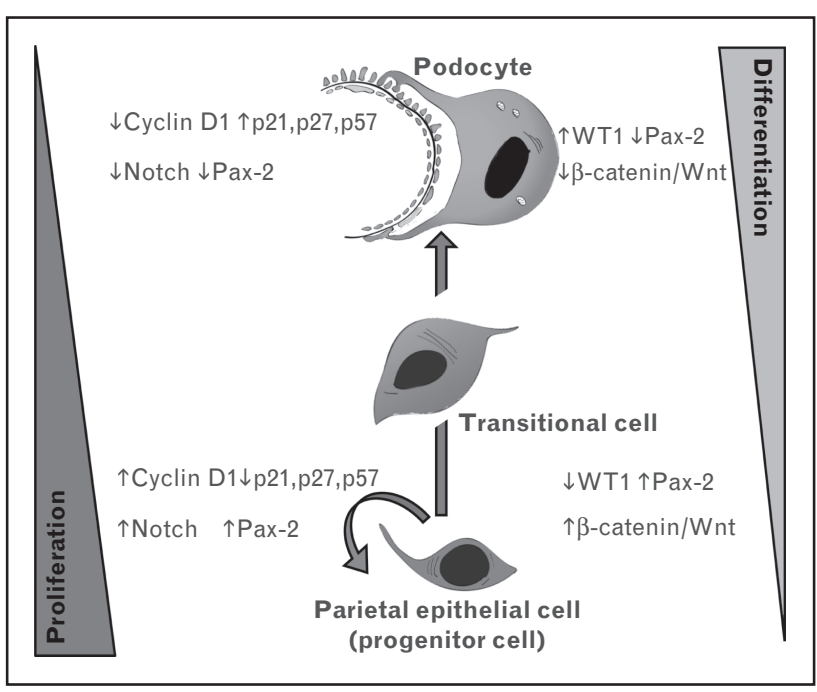

FIGURE 1. Signaling pathways regulating parietal epithelial cell proliferation and their differentiation toward podocytes. Schematic representation showing the signaling pathways involved in parietal epithelial cell (PEC) proliferation and how they are regulated when PECs are pushed to differentiate toward the podocyte phenotype. In particular, PECs are pushed to proliferate by high levels of Pax-2, Notch-2, and cyclin DI and by the absence of the cell-cycle inhibitors p21, p27, and p57. High levels of Pax-2 also suppress WT1 expression, which in turn allows activation of the $\beta$-catenin/Wnt pathway, keeping PECs in an undifferentiated state. When PECs downregulate Pax-2, this allows WT1 upregulation, which suppresses the $\beta$-catenin/ Wnt pathway that in turn induces PEC differentiation into podocytes. Differentiation of PECs into podocytes also associates with upregulation of the cell-cycle inhibitors p21, p27, p57 and suppression of cyclin D1 and of the Notch pathway, which makes the podocyte unable to proliferate.

$\beta$-catenin/Wnt signaling, which represents a pivotal step driving PEC differentiation into podocytes during kidney development [7]. Indeed, conditional $\beta$-catenin-knockout mice in which Ctnnb1 (mouse $\beta$-catenin gene) was removed during later stages of nephrogenesis in PECs show underdeveloped glomerular capillary tufts, with podocytes replacing normal flat PECs. These events demonstrate that $\beta$-catenin/Wnt signaling is required for proper differentiation and maturation of PECs into podocytes [8] (Fig. 1).

Interestingly, the $\beta$-catenin/Wnt signaling is also a direct activator of cyclin D1, a critical initiator of cell division, which controls PEC proliferation during development as well as postnatally. Starting at the $S$ phase of glomerulogenesis, the Src-suppressed protein kinase C substrate (SSeCKS), a multivalent scaffolding A kinase anchoring protein which sequesters cyclin D1 in the cytoplasm, is induced in embryonic PECs, but not in embryonic podocytes, and is constitutively expressed postnatally by PECs, but not podocytes, in normal glomeruli [9"] (Fig. 1). Phosphorylation of SSeCKS by activated protein kinase $C$ disrupts binding, resulting in nuclear translocation of cyclin D1 [9"]. Consistently, SSeCKS null mice show hyperplasia of PECs in otherwise normal glomeruli and develop significantly worse proteinuric glomerular disease, marked by increased PEC proliferation and expression of nuclear cyclin D1 [9"]. These observations suggest that PECs represent podocyte progenitors that are maintaining a modulated capacity to divide until they are in an undifferentiated state [10]. Although PECs exhibit a simple cytoskeleton and a high proliferative capacity, the highly specialized podocyte phenotype requires the acquisition of a complex cytoskeleton structure that would be disrupted to assemble the mitotic spindle, making it difficult to efficiently complete cytokinesis [10]. Consistently, in PECs, Notch activation triggers proliferation by promoting entry into the S-phase of the cell cycle and mitotic division (Fig. 1) [11]. However, attenuation of Notch activity is required to allow PECs to differentiate into podocytes, as demonstrated by the observation that an impaired downregulation of the Notch pathway induces generation of podocytes with an abnormal DNA content and their following death by mitotic catastrophe [11].

In summary, PECs represent progenitors for podocytes during normal kidney development, displaying the property to proliferate and amplify, a capacity that no longer exists once PECs are committed to differentiate into podocytes $[8,12]$. Like other progenitor cells [13], this process is regulated through an interaction among the $\beta$-catenin/Wnt signaling pathway and the Notch pathway $[11,14]$, which regulate PEC shift from entry within the cell cycle and proliferation to exit from the cell cycle and differentiation into podocytes (Fig. 1).

\section{Role of parietal epithelial cells as progenitor cells to enhance podocyte number}

The capacity of mature PECs to proliferate and differentiate into podocytes beyond glomerular development has been recently described in adult human, mouse, and rat kidney $[4,12,15,16]$. In humans, PECs represent a heterogeneous population of progenitors, characterized by the coexpression of two surface markers, CD24 and CD133, used to identify human stem or progenitor cells in several adult human tissues $[17,18]$. PECs localized to the urinary pole that express progenitor, but not podocyte, markers can differentiate into tubular cells, as well as podocytes [16]. By contrast, a subpopulation of PECs localized along the Bowman's capsule expressing both progenitor and podocyte markers 
regenerate only into podocytes [16]. Finally, cells localized to the vascular pole do not normally express progenitor markers, but display phenotypic features of fully differentiated podocytes [16]. In contrast to other kidney cells, PECs expressing $\mathrm{CD} 24^{+} \mathrm{CD} 133^{+}$also express CD106. This expression profile distinguishes them from other $\mathrm{CD} 24^{+} \mathrm{CD} 133^{+}$cells scattered along the tubule, displaying phenotypic and functional features of tubular progenitors [19]. Although they only partially share markers with human cells, PECs with similar progenitor features and anatomical localization were also identified in mouse and rat kidneys $\left[4,20^{\text {"}}\right]$. Indeed, cells with features of both PECs and podocytes could be detected at the glomerular vascular stalk in mouse [4].

The elegant genetic tagging of PECs in a tripletransgenic doxycycline-inducible mouse line demonstrated that podocytes are recruited from PECs, which proliferate and differentiate from the urinary to the vascular stalk, generating new podocytes [4]. In addition, in adult rat kidneys, three distinct cell populations of PECs lining the rat Bowman's capsule have been identified, including the most abundant one of immature progenitor cells, transitional cells expressing markers for both progenitor cells and podocytes, and a subset of differentiated cells, the parietal podocytes [20"]. Recently, several studies suggested the possibility that PECs also migrate from Bowman's capsule to the capillary tuft in regions different from the vascular pole [21]. Interestingly, the possibility of PECs differentiating into podocytes in response to injury was recently hypothesized also in adult zebrafish [22]. PECs begin to express podocyte proteins in states of progressive podocyte depletion, including experimental focal segmental glomerulosclerosis (FSGS), membranous nephropathy, and aging nephropathy $[23,24]$.

\section{Role of parietal epithelial cells and filtered albumin}

Adjacent PECs adherent to the underlying Bowman's basement membrane exhibit tight junctions, and express the tight junction proteins claudin-1, occludin, and ZO-1 in normal rat, mouse, and human glomeruli [25]. Tight junctions are disrupted in experimental anti-glomerular basement membrane (GBM) disease, accompanied by reduced levels of these tight junction proteins [25]. In an attempt to determine the biological consequences of these changes, an in-vivo permeability assay was performed using different-sized labeled tracers. Following direct podocyte injury leading to proteinuria, the permeability to tracers similar in size to albumin was increased between adjacent
PECs. This leads to tracers leaking from the urinary space into the periglomerular compartment [25]. The consequences of this remain unclear, although one might speculate that the presence of glomerular filtrate in the periglomerular space might be proinflammatory.

Under normal conditions, PECs in culture and PECs in vivo take up small amounts of albumin. However, when cultured PECs are exposed to increased albumin concentrations, albumin abundance increases, in part due to endocytosis. Increased intracellular albumin leads to apoptosis [26]. Similarly, PECs in vivo also show increased intracellular levels in experimental models of proteinuria, accompanied by apoptosis. Taken together, through tight junctions and endocytosis, PECs might normally play an important role in filtered albumin.

\section{PARIETAL EPITHELIAL CELLS AS THE BAD GUYS IN DISEASE}

Although the data discussed above is consistent with PECs portrayed as vital cells for normal development and glomerular repair, they paradoxically also have a 'bad side' to them in certain disease states, in which their responses directly contribute to glomerular disease and, in certain circumstances, deterioration in glomerular function.

\section{Parietal epithelial cell proliferation: role in crescentic glomerulonephritis and collapsing glomerulopathy}

To date, there are no glomerular diseases considered 'PEC-specific'. Crescentic glomerulonephritis is perhaps the best-known disease in which PECs contribute significantly to the kidney disease, although the initial injury likely arises in the glomerular endothelial cells, podocytes, and/or GBM. The cellular crescent is a unique glomerular lesion that implies glomerular epithelial cell overgrowth to fill-up Bowman's space that compromises glomerular function. There has been a debate about the type of epithelial cells inside crescents, because crescentic lesions often stain positive for both PEC and podocyte markers $[27,28]$. However, elegant lineage tracing studies in mice have finally demonstrated clearly that PEC proliferation leads to marked increase in cell number within crescents of murine nephrotoxic serum nephritis and collapsing glomerulopathy [29]. The concept that the low mitotic activity of terminally differentiated podocytes could increase via 'dedifferentiation' to podocyte hyperplasia-related crescents, which was before solely based on immunostaining, was finally disproved by these lineage-tracing studies [29]. In hindsight, 
some of the confusion related to cells in crescents staining positive for podocyte markers is rather due to the expression of such proteins by activated PECs [23]. In addition, podocytes sometimes stain positive for proliferation markers like Ki67 or BrdU, but this indicates cell-cycle entry and not necessarily podocyte mitosis. During glomerular injury, podocytes certainly enter the S-phase of the cell cycle to undergo hypertrophy, whereas when being forced to override the $\mathrm{G}_{2} / \mathrm{M}$ check point of the cell cycle, they usually undergo an aberrant mitosis leading to podocyte detachment and death, that is, mitotic catastrophe $[10,11]$. Finally, studies on human renal biopsy material are consistent with the concept that it is the PEC overgrowth that accounts for epithelial hyperplasia in crescentic and collapsing glomerulopathies [30].

What factors/pathways drive the mitotic activity of PECs in these disorders? Crescent formation most frequently occurs in necrotizing and highly inflammatory forms of glomerulonephritis, which has raised the concept that it is the inflammatory component that accounts for crescent formation. However, crescents develop in multiple other glomerulopathies including late-stage glomerulosclerosis and non-inflammatory glomerulopathies. The group led by Moeller showed that in a transgenic mouse model of selective PEC depletion with diphtheria toxin, PEC necrosis leading to loss is sufficient to drive crescent formation. This is most likely due to an 'overcompensatory' epithelial repair response, driven by surviving PECs [31]. Even though this study proves the PEC's capacity to create hyperplastic lesions and crescents upon injury, selective PEC necrosis is not a typical feature of common crescentic glomerulopathies, which, rather, display necrotic lesions of the glomerular tuft. Tuft necrosis involves glomerular vascular injury, and accumulating evidence suggests that plasma leakage provides mitogenic stimuli that drive PEC hyperplasia and crescent formation. PECs (like other epithelial cells) usually live in an environment devoid of plasma exposure. The rupture of glomerular capillaries in crescentic disease increases the plasma concentration inside Bowman's space by 20-40\%. Experimentally, this milieu dramatically increases the proliferation of murine and human PECs in culture [32"]. Several plasma components could account for this PEC hyperplasia but, currently, there are consistent data only for fibrinogen activation, an element of the activated coagulation cascade during vascular injuries. A lack of fibrinogen or fibrinolysis prevents PEC hyperplasia formation in several rodent models of glomerular crescent formation [32",33]. PDGF-D, CXCR4, and epidermal growth factor receptor (EGFR) signaling is also associated with crescent formation $[34,35]$, but the related evidence originates from experimental overexpression, which does not mirror physiological conditions. In addition, in these studies, the transgene was overexpressed in podocytes, so PECs were only indirectly affected. Angiotensin-converting enzyme (ACE) inhibition helps to reduce crescent formation in rodents $\left[20^{-}, 32^{-}\right]$, but it remains unclear whether this relates to the hemodynamic or the paracrine effects of angiotensin II.

Cellular crescents can progress to fibrocellular crescents, due to the accumulation of extracellular, and potentially irreversible, matrix deposits that predict a poor renal outcome. This process might be the result of PEC epithelial-to-mesenchymal transition, characterized by a loss of epithelial polarity and increased secretion of extracellular matrix components. The loss of polarity becomes obvious by the fact that PECs that undergo this process secrete extracellular matrix to not only their basal aspect but also all around like mesenchymal cells, which creates the honeycomb-like lesions typical for fibrocellular crescents [32"].

Another glomerulopathy that was recently related to an abnormal PEC proliferation hyperplasia is collapsing glomerulopathy. Collapsing glomerulopathy is characterized histologically by segmental to global collapse of the capillary tuft and pronounced epithelial cell hyperplasia, often referred as pseudo-crescents. Lineage tracing studies demonstrated that these monolayer lesions located directly on top of the glomerular convolute consisted of genetically tagged PECs that proliferate in a dysregulated manner in response to massive podocyte injury, further suggesting that the type of PEC response to podocyte injury is an important driver of progression toward glomerulosclerosis.

\section{Parietal epithelial cells and glomerular scarring}

FSGS, characterized by scarring in a segment of glomeruli, has traditionally been considered primarily a disease of podocytes. One of the earliest hallmarks of classic FSGS is the formation of a synechial attachment, in which the glomerular tuft and Bowman's capsule become adherent. The traditional view has been that the segmental scarring was a consequence of injured or damaged podocytes, as these cells have the ability to produce a variety of extracellular matrix proteins. However, a role for PECs in this sclerotic process has emerged over recent years as follows. In the era predating currently used cell fate mapping techniques, several well performed studies using immunostaining for cell 'specific' markers showed the presence of PECs 
within sclerotic areas in experimental and human disease $[36,37]$. These studies suggested that PECs might participate in the focal sclerotic glomerular process.

Subsequently, utilizing PEC-reporter mice, Smeets et al. [38"'] have provided convincing data for the role of PECs producing matrix proteins, and thereby significantly contributing to the segmental scarring process. They showed that following a primary injury to podocytes in these mice, PECs become 'activated' as denoted by the de-novo expression of CD44. In this activated state, PECs begin to produce extracellular matrix proteins, which initially accumulate along Bowman's basement membrane, leading to thickening of this structure. They also showed that activated PECs can later be detected on the glomerular tuft, where they continue to produce Bowman's capsule type matrix [38"']. Similar observations were reported also in human biopsies [39"']. The continuum of newly made Bowman's capsule-type matrix by PECs at these two adjacent sites ultimately leads to a 'bridge' of matrix that extends from Bowman's basement membrane to the glomerular tuft, comprising the characteristic fibrous attachment/ adhesion of these two structures in FSGS. As more PECs invade the glomerular tuft, extracellular matrix proteins continually and progressively accumulate beyond this original site, ultimately leading to an advanced sclerotic lesion of FSGS. More recently, transcriptomic studies add further evidence for a role of PECs in matrix accumulation, wherein an abundance of genes considered 'PEC-specific' were detected in areas of sclerosis in human glomeruli [40]. Thus, one current view underlying the mechanism of segmental scarring in FSGS is that the extracellular matrix accumulation likely arises from two sources: injured podocytes and activated PECs. Definitive causeand-effect studies are still needed.

A recent study in human glomerular diseases showed that similar to experimental conditions of FSGS, PECs begin to express de novo CD44, a glycoprotein involved in cell adhesion, cell matrix interaction, and cell migration [29,31,38"']. Noteworthy was that in human biopsies from patients with minimal change disease (also a podocyte disease), CD44 was barely expressed [39"']. Although the precise biological consequence of increased CD44 expression in PECs is unclear, CD44 staining might serve as a useful 'marker' to distinguish between FSGS and minimal change disease in kidney biopsies. Finally, PECs (in addition to podocytes) have also been detected in the urine of patients with active FSGS [41], although the consequences of this too remain unclear.

\section{Parietal epithelial cells as a potential therapeutic target in disease}

Like most kidney cells, there are no reports on cellspecific drug targets in PECs. However, the discovery that adult PECs can differentiate into podocytes provides hope that this pathway might be pharmacologically modulated in order to enhance new podocyte formation. Several reports show that PECs are responsive to certain therapeutics. ACE inhibition reduces PEC proliferation in experimental human immune deficiency associated nephropathy (HIVAN) [42], and ACE inhibition has also been demonstrated to enhance the capacity of PECs to become progenitor cells [20"]. Zhang et al. [43"] showed that administering retinoids to animals with experimental FSGS or membranous nephropathy augmented the number of PECs that express podocyte proteins, suggesting that under these circumstances, retinoids might enhance the progenitor capacity of PECs. The precise role for vitamin D in PEC biology is unknown, but might be of importance, as the vitamin D receptor is expressed on PECs [44], and vitamin D is required for the differentiation of PECs toward podocytes in culture [45]. In addition, it was recently demonstrated that glomerular regeneration could be modulated using Notch inhibitors in mouse models of FSGS, thus directly influencing the amount of proteinuria and the occurrence of glomerulosclerosis [11]. Recent studies suggest that blockade of the chemokine stromal derived factor-1 (SDF-1/CXCL12) also increases podocyte number, reduces proteinuria, and ameliorates renal dysfunction in nephropathy of type 2 diabetic mice by enhancing PEC differentiation toward the podocyte phenotype [46,47]. Indeed, CXCL12 blockade increased renal mRNA expression levels of nephrin and podocin, two podocyte-slit membrane-related proteins that serve as markers of podocyte differentiation [47], further suggesting that pharmacological treatments can be used to modulate PEC growth and/or differentiation into podocytes. Other potential therapeutic targets for PECs in disease might include epidermal growth factor [48] and its receptor [49], as well as the amino acid transporter LAT2 [50]. More recently, Ueno et al. [51] showed that inhibiting Notch in vivo in experimental collapsing FSGS reduced the expression of mesenchymal-like markers including alpha smooth muscle actin, vimentin, and snail.

\section{CONCLUSION}

Our understanding of the biology and pathophysiology of PECs is increasing substantially, as it relates to how these glomerular epithelial cells might be reparative on the one hand, yet injurious on the 
other hand [52]. Many tools are being developed to get us to the next level of understanding on what triggers and regulates these processes, and which molecular targets we might want to alter to hasten repair and reduce progression of glomerular disease.

\section{Acknowledgements}

H.J.A. is supported by a grant from the Deutsche Forschungsgemeinschaft (AN372/15-1). S.J.S. is supported by NIH grants. P.R. is supported by the European Community under the European Community's Seventh Framework Programme (FP7/2012-2016; grant number 305436).

\section{Conflicts of interest}

There are no conflicts of interest.

\section{REFERENCES AND RECOMMENDED READING}

Papers of particular interest, published within the annual period of review, have been highlighted as:

- of special interest

a. of outstanding interest

Additional references related to this topic can also be found in the Current

World Literature section in this issue (p. 362).

1. Ohse T, Pippin JW, Chang AM, et al. The enigmatic parietal epithelial cell is finally getting noticed: a review. Kidney Int 2009; 76:1225-1238.

2. Ohse T, Pippin JW, Vaughan MR, et al. Establishment of conditionally immortalized mouse glomerular parietal epithelial cells in culture. J Am Soc Nephrol 2008; 19:1879-1890.

3. Kabgani N, Grigoleit T, Schulte K, et al. Primary cultures of glomerular parietal epithelial cells or podocytes with proven origin. PLoS One 2012; 7:e34907.

4. Appel D, Kershaw DB, Smeets B, et al. Recruitment of podocytes from glomerular parietal epithelial cells. J Am Soc Nephrol 2009; 20:333-343.

5. Romagnani P. Toward the identification of a 'renopoietic system'. Stem Cells 2009; 27:2247-2253.

6. Wagner KD, Wagner $\mathrm{N}$, Guo JK, et al. An inducible mouse model for PAX2-dependent glomerular disease: insights into a complex pathogenesis. Curr Biol 2006; 16:793-800.

7. Kim MK, McGarry TJ, O Broin $P$, et al. An integrated genome screen identifies the Wnt signaling pathway as a major target of WT1. Proc Natl Acad Sci U S A 2009; 106:11154-11159.

8. Grouls S, Iglesias DM, Wentzensen N, et al. Lineage specification of parietal epithelial cells requires beta-catenin/Wnt signaling. J Am Soc Nephrol 2012; 23:63-72.

9. Burnworth B, Pippin J, Karna $P$, et al. SSeCKS sequesters cyclin D1 in

- glomerular parietal epithelial cells and influences proliferative injury in the glomerulus. Lab Invest 2012; 92:499-510.

The data show that SSeCKS, a multivalent scaffolding A kinase anchoring protein, sequesters cyclin D1 in the cytoplasm of quiescent PECs. Cyclin D1 was immunoprecipitated with SSeCKS from capsulated glomeruli containing PECs, whereas decapsulated glomeruli without PECs lacked SSeCKS and cyclin D1. SSeCKS null mice showed hyperplasia of PECs in otherwise normal glomeruli and developed significantly worse proteinuric glomerular disease, marked by increased PEC proliferation and expression of nuclear cyclin D1, from nephrotoxic nephritis. These results suggest that SSeCKS controls the localization and activity of cyclin D1 in PECs and influences proliferative injury in the glomerulus.

10. Lasagni L, Lazzeri E, Shankland SJ, et al. Podocyte mitosis: a catastrophe. Curr Mol Med 2013; 13:13-23.

11. Lasagni $L$, Ballerini $L$, Angelotti $M L$, et al. Notch activation differentially regulates renal progenitors proliferation and differentiation toward the podocyte lineage in glomerular disorders. Stem Cells 2010; 28:16741685.

12. Lazzeri $E$, Crescioli $C$, Ronconi $E$, et al. Regenerative potential of embryonic renal multipotent progenitors in acute renal failure. J Am Soc Nephrol 2007; 18:3128-3138.

13. Nakamura $T$, Tsuchiya $K$, Watanabe M. Crosstalk between Wnt and Notch signaling in intestinal epithelial cell fate decision. J Gastroenterol 2007; 42:705-710.

14. Grouls $S$, Iglesias DM, Wentzensen $N$, et al. Lineage specification of parietal epithelial cells requires $\{$ beta\}-catenin/Wnt signaling. J Am Soc Nephrol 2012; 23:63-72.
15. Sagrinati $C$, Netti GS, Mazzinghi $B$, et al. Isolation and characterization of multipotent progenitor cells from the Bowman's capsule of adult human kidneys. J Am Soc Nephrol 2006; 17:2443-2456.

16. Ronconi E, Sagrinati C, Angelotti ML, et al. Regeneration of glomerular podocytes by human renal progenitors. J Am Soc Nephrol 2009; 20:322332.

17. Lasagni L, Romagnani P. Glomerular epithelial stem cells: the good, the bad, and the ugly. J Am Soc Nephrol 2010; 21:1612-1619.

18. Romagnani $P$, Remuzzi $G$. Renal progenitors in non-diabetic and diabetic nephropathies. Trends Endocrinol Metab 2013; 24:13-20.

19. Angelotti ML, Ronconi E, Ballerini L, et al. Characterization of renal progenitors committed toward tubular lineage and their regenerative potential in renal tubular injury. Stem Cells 2012; 30:1714-1725.

20. Benigni A, Morigi M, Rizzo $P$, et al. Inhibiting angiotensin-converting

- enzyme promotes renal repair by limiting progenitor cell proliferation and restoring the glomerular architecture. Am J Pathol 2011; 179:628638.

Three distinct populations of PECs were identified in the rat Bowman's capsule. After exposure to inductive medium, cultured PECs that were obtained by capsulated glomeruli generated podocytes, documenting their progenitor nature. Mitotic activity of cultured renal progenitors was induced by angiotensin II through the downregulation of cell-cycle inhibitor C/EBP expression.

21. Peti-Peterdi J, Sipos A. A high-powered view of the filtration barrier. J Am Soc Nephrol 2010; 21:1835-1841.

22. Zhou W, Hildebrandt F. Inducible podocyte injury and proteinuria in transgenic zebrafish. J Am Soc Nephrol 2012; 23:1039-1047.

23. Ohse $T$, Vaughan MR, Kopp JB, et al. De novo expression of podocyte proteins in parietal epithelial cells during experimental glomerular disease. Am J Physiol Renal Physiol 2010; 298:F702-F711.

24. Zhang J, Hansen KM, Pippin JW, et al. De novo expression of podocyte proteins in parietal epithelial cells in experimental aging nephropathy. Am J Physiol Renal Physiol 2012; 302:F571-F580.

25. Ohse T, Chang AM, Pippin JW, et al. A new function for parietal epithelial cells: a second glomerular barrier. Am J Physiol Renal Physiol 2009; 297:F1566-F1574.

26. Chang AM, Ohse T, Krofft RD, et al. Albumin-induced apoptosis of glomerular parietal epithelial cells is modulated by extracellular signal-regulated kinase 1/2. Nephrol Dial Transplant 2012; 27:1330-1343.

27. Singh SK, Jeansson $M$, Quaggin SE. New insights into the pathogenesis of cellular crescents. Curr Opin Nephrol Hypertens 2011; 20:258-262.

28. Smeets $B$, Moeller MJ. Parietal epithelial cells and podocytes in glomerular diseases. Semin Nephrol 2012; 32:357-367.

29. Smeets $B$, Uhlig S, Fuss $A$, et al. Tracing the origin of glomerular extracapillary lesions from parietal epithelial cells. J Am Soc Nephrol 2009; 20:26042615.

30. Smeets B, Angelotti ML, Rizzo P, et al. Renal progenitor cells contribute to hyperplastic lesions of podocytopathies and crescentic glomerulonephritis. J Am Soc Nephrol 2009; 20:2593-2603.

31. Sicking EM, Fuss A, Uhlig S, et al. Subtotal ablation of parietal epithelial cells induces crescent formation. J Am Soc Nephrol 2012; 23:629640.

32. Ryu M, Migliorini $A$, Miosge $N$, et al. Plasma leakage through glomerular

- basement membrane ruptures triggers the proliferation of parietal epithelial cells and crescent formation in non-inflammatory glomerular injury. J Pathol 2012. doi: 10.1002/path.4046. [Epub ahead of print]

The data from experimental and human Alport show that glomerular vascular injury and GBM breaks cause plasma leakage, which triggers a wound healing program involving the proliferation of parietal cells and their loss of polarity. This process can trigger cellular and fibrocellular crescent formation even in the absence of cellular inflammation and rupture of the Bowman's capsule.

33. Drew AF, Tucker HL, Liu H, et al. Crescentic glomerulonephritis is diminished in fibrinogen-deficient mice. Am J Physiol Renal Physiol 2001; 281:F1157F1163.

34. van Roeyen CR, Eitner F, Boor $\mathrm{P}$, et al. Induction of progressive glomerulonephritis by podocyte-specific overexpression of platelet-derived growth factor-D. Kidney Int 2011; 80:1292-1305.

35. Ding M, Cui S, Li C, et al. Loss of the tumor suppressor Vhlh leads to upregulation of $\mathrm{Cxcr} 4$ and rapidly progressive glomerulonephritis in mice. Nat Med 2006; 12:1081-1087.

36. Ohtaka A, Ootaka $T$, Sato $H$, et al. Significance of early phenotypic change of glomerular podocytes detected by Pax2 in primary focal segmental glomerulosclerosis. Am J Kidney Dis 2002; 39:475-485.

37. Nagata $M$, Hattori $M$, Hamano $Y$, et al. Origin and phenotypic features of hyperplastic epithelial cells in collapsing glomerulopathy. Am J Kidney Dis 1998; 32:962-969

38. Smeets B, Kuppe C, Sicking EM, et al. Parietal epithelial cells participate in

a. the formation of sclerotic lesions in focal segmental glomerulosclerosis. J Am Soc Nephrol 2011; 22:1262-1274.

Using a PEC reporter mouse, the authors show that in a model of experimental glomerular disease, PECs are activated and produce increased extracellular matrix proteins. This forms the backbone of the synechial attachment of FSGS. When activated PECs migrate to the glomerular tuft, they lead to segmental glomerular scarring. 
39. Fatima $\mathrm{H}$, Moeller MJ, Smeets $\mathrm{B}$, et al. Parietal epithelial cell activation marker -1. in early recurrence of FSGS in the transplant. Clin J Am Soc Nephrol 2012; $7: 1852-1858$

CD44 is a marker of activated PECs. The authors show that CD44 staining is detected in patients with recurrent FSGS, but not minimal change disease. CD44 may, therefore, serve as a useful marker to distinguish these podocyte diseases.

40. Hodgin JB, Borczuk AC, Nasr SH, et al. A molecular profile of focal segmental glomerulosclerosis from formalin-fixed, paraffin-embedded tissue. Am J Pathol 2010; 177:1674-1686.

41. Achenbach J, Mengel M, Tossidou I, et al. Parietal epithelia cells in the urine as a marker of disease activity in glomerular diseases. Nephrol Dial Transplant 2008; 23:3138-3145.

42. Yadav A, Vallabu S, Kumar D, et al. HIVAN phenotype: consequence of epithelial mesenchymal transdifferentiation. Am J Physiol Renal Physiol 2010; 298:F734-744.

43. Zhang J, Pippin JW, Vaughan MR, et al. Retinoids augment the expression of

- podocyte proteins by glomerular parietal epithelial cells in experimental glomerular disease. Nephron Exp Nephrol 2012; 121:e23-37.

In experimental membranous nephropathy and experimental FSGS, the authors show that the improvement in proteinuria, glomerulosclerosis, and podocyte number following the administration of retinoids correlates with an increase in the number of PEC progenitors.

44. Wang $Y$, Borchert $M L$, Deluca HF. Identification of the vitamin $D$ receptor in various cells of the mouse kidney. Kidney Int 2012; 81:9931001.
45. Smeets $B$, Angelotti ML, Rizzo $P$, et al. Renal progenitor cells contribute to hyperplastic lesions of podocytopathies and crescentic glomerulonephritis. J Am Soc Nephrol 2009; 20:2593-2603.

46. Sayyed SG, Hagele H, Kulkarni OP, et al. Podocytes produce homeostatic chemokine stromal cell-derived factor-1/CXCL12, which contributes to glomerulosclerosis, podocyte loss and albuminuria in a mouse model of type 2 diabetes. Diabetologia 2009; 52:2445-2454.

47. Darisipudi MN, Kulkarni OP, Sayyed SG, et al. Dual blockade of the homeostatic chemokine CXCL12 and the proinflammatory chemokine CCL2 has additive protective effects on diabetic kidney disease. Am J Pathol 2011; 179:116-124.

48. Flamant $\mathrm{M}$, Bollee $\mathrm{G}$, Henique $\mathrm{C}$, Tharaux $\mathrm{PL}$. Epidermal growth factor: a new therapeutic target in glomerular disease. Nephrol Dial Transplant 2012; 27:1297-1304.

49. Bollee G, Flamant M, Schordan S, et al. Epidermal growth factor receptor promotes glomerular injury and renal failure in rapidly progressive crescentic glomerulonephritis. Nat Med 2011; 17:1242-1250.

50. Kurayama R, Ito N, Nishibori $\mathrm{Y}$, et al. Role of amino acid transporter LAT2 in the activation of mTORC1 pathway and the pathogenesis of crescentic glomerulonephritis. Lab Invest 2011; 91:992-1006.

51. Ueno $T$, Kobayashi $N$, Nakayama $M$, et al. Aberrant Notch1-dependent migration and dedifferentiation of glomerular parietal epithelial cells promotes collapsing focal segmental glomerulosclerosis with progressive podocyte loss. Kidney Int (in press).

52. Romagnani $P$, Lasagni $L$, Remuzzi $G$. Renal progenitors: an evolutionary conserved strategy for kidney regeneration. Nat Rev Nephrol 2013; 9:137-146. 